\title{
SINCRONISMO EM REDES MESTRE-ESCRAVO COM ATRASO
}

\author{
Luiz Henrique Alves Monteiro ${ }^{\dagger *}$ \\ Paulo Alves Garcia ${ }^{\dagger}$
}

José Roberto Castilho Piqueira*

*Universidade de São Paulo, Escola Politécnica - Departamento de Telecomunicações e Controle Av. Prof. Luciano Gualberto, travessa 3, 158, CEP 05508-900 - São Paulo, SP, Brasil

†Universidade Presbiteriana Mackenzie, Pós-graduação - Engenharia Elétrica, Rua da Consolação, 896 Edifício Amantino Vassão, térreo, CEP 01302-907 - São Paulo, SP, Brasil

\begin{abstract}
The dynamical behavior of synchronous telecommunication networks under slow changes caused by degradation in the oscillators composing the network is studied. These oscillators are phase-locked loops (PLLs). Through the Dynamical System Theory, the behaviors of a single second-order PLL and of such a PLL connected in master-slave single chain network and masterslave single loop networks are analysed. The delay on signal propagation between the oscillators is taken into account. The influence of the parameter values of the oscillators on the existence and on the stability of the synchronous solution for networks composed by one master and one slave is discussed. In the chain network, the delay forbids the existence of synchronous solution; in the single loop network; such a solution only can exist when the propagation time of the signal from the master to the slave is equal to that from the slave to the master.
\end{abstract}

KEYWORDS: phase-locked loop, master-slave network, synchronism, bifurcation

Artigo submetido em 10/10/01

1a. Revisão em 26/12/01; 2a. Revisão em 12/07/02

Aceito sob recomendação do Ed. Assoc. Prof. Takashi Yoneyama

\section{RESUMO}

Estuda-se o comportamento dinâmico de redes de telecomunicações síncronas, frente a variações lentas causadas pela degradação dos osciladores que compõem a rede. Esses osciladores são malhas de sincronismo de fase ("phase-locked loops" - PLLs). Através da Teoria de Sistemas Dinâmicos, analisam-se os comportamentos de um PLL de segunda ordem isolado e desse PLL formando redes mestre-escravo do tipo cadeia simples e do tipo enlace simples. O atraso na propagação do sinal entre os osciladores é levado em consideração. Discute-se a influência dos valores dos parâmetros dos osciladores na existência e na estabilidade da solução síncrona, para redes constituídas de um mestre e um escravo. Na rede cadeia simples, o atraso impede a existência de solução síncrona; na rede enlace simples, tal solução só pode existir quando são iguais os tempos de propagação do sinal do mestre para o escravo e do escravo para o mestre.

PALAVRAS-CHAVE: malha de sincronismo de fase, rede mestre-escravo, sincronismo, bifurcação

\section{INTRODUÇÃO}

$\mathrm{Na}$ natureza, encontram-se vários exemplos de sincronismo em redes de osciladores. Exemplos clássicos são: 
neurônios que controlam movimentos periódicos, como aqueles necessários para a natação (Cohen et alli, 1982); neurônios relacionados a processos cognitivos (Engel et alli, 1992); células cardíacas do nó sino-atrial que geram impulsos elétricos (Bernardo et alli, 1998). Sistemas formados por osciladores, que se acoplam e se influenciam, não precisam estar, necessariamente, confinados num único organismo. Sincronizam-se também: vaga-lumes machos que piscam simultaneamente para atraírem as fêmeas da vizinhança (Buck e Buck, 1976); grilos que cricrilam em uníssono num ritual de acasalamento (Walker, 1969); o ciclo menstrual de mulheres confinadas num internato (McClintock, 1971); o aplauso da platéia em salas de concerto húngaras (Néda et alli, 2000).

Sincronismo é assunto de interesse para o projetista de redes de telecomunicações. Quanto à estratégia usada para se obter a sincronização, as redes de telecomunicações podem ser divididas em duas categorias. Nas redes plesiócronas, são utilizados relógios independentes, extremamente precisos, com desvios de freqüência muito pequenos. A vantagem dessa estratégia é a robustez a falhas, já que a falha de um nó não acarreta a falha de outros nós. A desvantagem está no alto custo e na necessidade de se realizarem ajustes periódicos. Nas redes síncronas, o sincronismo é estabelecido e mantido através da troca de sinais de controle entre os relógios que compõem a rede. A vantagem dessa estratégia está no custo menor, se comparado com o custo de uma rede plesiócrona. A desvantagem está no fato de que a falha de um relógio pode comprometer o funcionamento de outros (Lindsey et alli, 1985).

As redes síncronas podem ser divididas em duas categorias, quanto à natureza do sinal de controle. Nas redes mutuamente sincronizadas, cada nó recebe um sinal de outros nós da rede, sendo o sincronismo atingido a partir de uma média ponderada desses sinais. Eventuais reconfigurações da rede podem tornar instável a solução síncrona original. Nas redes do tipo mestre-escravo, o sinal de sincronismo é gerado a partir do relógio mestre, normalmente caro e preciso, e distribuído para os demais nós da rede. É claro que, quando há falha no mestre, compromete-se a geração do sinal de sincronismo. Perda de sincronismo leva à perda, parcial ou total, da informação transmitida. No caso de transmissão de informação criptografada, normalmente a perda é total.

Redes mestre-escravo são as mais utilizadas na prática (Lindsey et alli, 1985). Os primórdios dos problemas de sincronismo em redes mestre-escravo datam de meados da década de 60 do século passado (Gersho e Karafin, 1966; Karnaugh, 1966), quando as comunicações digitais começaram a adquirir importância e quando a comutação e a transmissão, digitais e integradas, foram associadas com efetividade, requerendo recuperação precisa de sinais digitais de relógio obtida a partir do sinal de linha, analógico por natureza. Na década de 80 , as propostas de redes síncronas, como SDH ("synchronous digital hierarchy") e SONET ("synchronous optical network") (Sexton e Reid, 1992), tornaram inevitável o estudo detalhado do problema, considerando possíveis não-linearidades e atrasos.

O objetivo deste trabalho é determinar as condições de sincronismo em redes mestre-escravo do tipo cadeia simples e do tipo enlace simples, levando-se em conta o atraso na propagação do sinal entre os osciladores. $\mathrm{Na}$ topologia cadeia simples, o sinal do mestre propaga-se em direção aos escravos, que formam uma fileira de osciladores encadeados. A topologia enlace simples é a topologia cadeia simples com uma única diferença: o sinal do último escravo é usado pelo mestre para realizar eventuais ajustes de freqüência. Aqui, analisam-se redes formadas por apenas um mestre e um escravo, usando a Teoria de Sistemas Dinâmicos (Guckenheimer e Holmes, 1983; Monteiro, 2002), e procuram-se as condições de existência de soluções síncronas assintoticamente estáveis. Tais soluções correspondem a pontos de equilíbrio dos sistemas dinâmicos que governam as redes. O oscilador escravo é tomado com uma malha de sincronismo de fase ("phase-locked loop" - PLL) de segunda ordem. Na próxima seção, descreve-se, sucintamente, o comportamento dinâmico de tal PLL.

\section{MALHA DE SINCRONISMO DE FASE}

PLL é um dispositivo eletrônico usado, desde de 1932, em aplicações que exigem controle automático de freqüência, como, por exemplo, em sistemas de navegação e rastreamento por satélites; em aparelhos receptores de TV; em processos de demodulação de sinais analógicos e digitais (Hseih e Hung, 1996; Piqueira, 1997; Garcia, 2000). Sua configuração básica consiste de um detetor de fases ("phase detector" - PD), um filtro passabaixas ("low-pass filter" - LPF), e um oscilador controlado por tensão ("voltage controlled oscillator" - VCO), conectados conforme ilustra a figura 1. A função da malha é anular a variação temporal da diferença entre a fase do sinal de entrada $V_{i}$ e a fase do sinal $V_{o}$ gerado pelo VCO.

O PD é um multiplicador de sinais, no qual se compara a fase $\phi_{o}(t)$ do sinal gerado pelo VCO com a fase $\phi_{i}(t)$ do sinal de entrada. Considerando que $V_{i}$ seja proporcional a $\operatorname{sen}\left(\phi_{i}(t)+\omega_{0} t\right)$ e $V_{o}$ seja proporcional a $\cos \left(\phi_{o}(t)+\omega_{0} t\right)$, a saída $V_{d}$ do PD torna-se proporcio- 


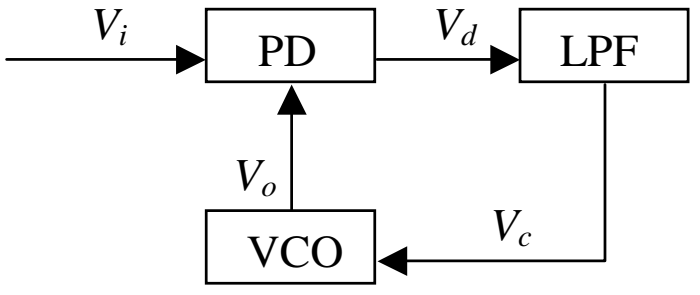

Figura 1: Diagrama de blocos de um PLL

nal a $\operatorname{sen}\left(\phi_{i}(t)-\phi_{o}(t)\right)+\operatorname{sen}\left(2 \omega_{0} t+\phi_{i}(t)+\phi_{o}(t)\right)$. Com essas escolhas para $V_{i}$ e $V_{o}$, assume-se que ambos possuem uma parte da variação temporal em comum (o termo $\left.\omega_{0} t\right)$; e que as eventuais diferenças são levadas em conta nas fases $\phi_{o}(t)$ e $\phi_{i}(t)$.

O sinal $V_{d}$ é aplicado ao LPF a fim de se eliminar o componente de alta freqüência (o termo $\operatorname{sen}\left(2 \omega_{0} t+\phi_{i}(t)+\right.$ $\left.\left.\phi_{o}(t)\right)\right)$. A saída $V_{c}$ do filtro alimenta o $\mathrm{VCO}$ e modifica a fase do seu sinal de acordo com a equação $d \phi_{o}(t) / d t$ $=k_{o} V_{c}$, sendo $k_{o}$ uma constante de proporcionalidade.

A equação diferencial que governa a dinâmica de um PLL é um grau maior do que a equação que caracteriza o filtro. Se o filtro é de primeira ordem, de modo que $V_{d}=V_{c}+\left(1 / \mu_{1}\right) V c^{\prime}$, então o PLL é governado pela equação de segunda ordem (Piqueira, 1997; Garcia, 2000, Monteiro 2002):

$$
\varphi^{\prime \prime}+\mu_{1} \varphi^{\prime}+\mu \mu_{1} \operatorname{sen} \varphi=F(t)
$$

sendo $\varphi(t) \equiv \phi_{i}(t)-\phi_{o}(t)$ o erro de fase entre o sinal de entrada e o sinal do VCO e $F(t) \equiv \phi_{i} "+\mu_{1} \phi_{i}^{\prime}$. Por simplicidade, usam-se as notações $x^{\prime} \equiv d x / d t$ e $x^{\prime \prime} \equiv$ $d^{2} x / d t^{2}$. O parâmetro $\mu_{1}=1 /(R C)$ é função da resistência $R$ e da capacitância $C$ do filtro. O parâmetro $\mu$ é proporcional a $k_{o}$ e ao ganho do detetor de fases. Repare que a equação (1) é estruturalmente equivalente à equação de um pêndulo linearmente amortecido, sujeito a uma função forçante $F(t)$. Essa equação pode ser reescrita como um sistema formado por duas equações diferenciais de primeira ordem, dadas por:

$$
\begin{gathered}
\varphi^{\prime}=\omega=f(\varphi, \omega) \\
\omega^{\prime}=F(t)-\mu_{1} \omega-\mu \mu_{1} \operatorname{sen} \varphi=g(\varphi, \omega)
\end{gathered}
$$

Esse sistema possui solução de equilíbrio estacionário somente se $F(t)=0$ ou $F(t)=$ constante $\equiv \mu_{1} \Omega$. Ou seja, para tais entradas, existem valores $\varphi^{*}$ e $\omega^{*}$ tais que $f\left(\varphi^{*}, \omega^{*}\right)=0$ e $g\left(\varphi^{*}, \omega^{*}\right)=0 . \quad F(t)=0$ representa o caso em que a fase do sinal de entrada $\phi_{i}(t)$ é constante; $F(t)=\mu_{1} \Omega$ representa a situação em que $\phi_{i}(t)$ cresce linearmente com o tempo, com velocidade $\Omega$ constante. O diagrama de bifurcações, ilustrado na figura 2 , resume

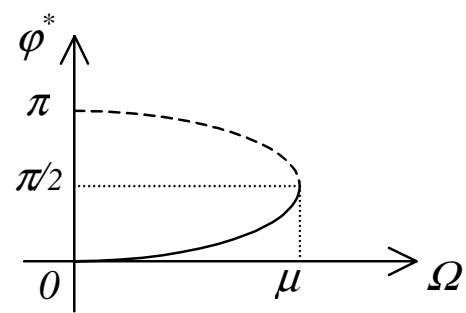

Figura 2: Diagrama de bifurcação

o comportamento assintótico do sistema dinâmico (2), tomando-se $F(t)=\mu_{1} \Omega$.

A estabilidade das soluções de equilíbrio, no sentido de Lyapunov, é determinada a partir do cálculo dos autovalores da matriz jacobiana associada. A primeira linha dessa matriz é formada por $\partial f / \partial \varphi$ e $\partial f / \partial \omega$; a segunda, por $\partial g / \partial \varphi$ e $\partial g / \partial \omega$; sendo que tais termos são calculados no ponto de equilíbrio $\left(\varphi^{*}, \omega^{*}\right)$.Se todos os autovalores dessa matriz têm parte real negativa, tem-se estabilidade assintótica; se há algum com parte real positiva, tem-se instabilidade.

Para o sistema (2), tem-se que quando $\Omega>\mu$, não há solução de equilíbrio $\varphi^{*}$; para $\Omega<\mu$ há duas. Como, para tal sistema, $\partial f / \partial \varphi=0, \partial f / \partial \omega=1, \partial g / \partial \varphi$ $=-\mu \mu_{1} \cos \varphi^{*}, \partial g / \partial \omega=-\mu_{1}$; então os autovalores $\lambda$ obedecem à equação característica $\lambda^{2}+\mu_{1} \lambda+$ $\mu \quad \mu_{1} \cos \varphi^{*}=0$. Para $\Omega<\mu$, o ponto de equilíbrio $\left(\varphi^{*}=\operatorname{arcsen}(\Omega / \mu), \omega^{*}=0\right)$ possui $\cos \varphi^{*}>0$; portanto, é assintoticamente estável (sendo representado por linha cheia na figura 2). Já o ponto $\left(\varphi^{*}=\pi-\right.$ $\left.\operatorname{arcsen}(\Omega / \mu), \omega^{*}=0\right)$ apresenta $\cos \varphi^{*}<0$,e é, conseqüentemente, instável (sendo representado por linha tracejada na figura 2). $\operatorname{Em} \Omega=\mu$, ocorre uma bifurcação sela-nó. O Teorema de Bendixson (Guckenheimer e Holmes, 1983; Monteiro, 2002) exclui a possibilidade de ciclo-limite, já que o divergente desse campo vetorial (dado por $\partial f / \partial \varphi+\partial g / \partial \omega=-1$ ) não muda de sinal em região alguma do espaço de fases $\varphi-\omega$. Assim, não são permitidas oscilações auto-sustentadas em $\varphi(t)$.

\section{REDES MESTRE-ESCRAVO CADEIA SIMPLES}

Considere uma rede composta por um mestre e um escravo em topologia cadeia simples, conforme ilustra a figura 3. Atribui-se o índice 1 ao nó mestre e o índice 2 ao nó escravo. O objetivo é estudar a existência e a estabilidade da solução síncrona com relação à variação nos parâmetros que expressam uma degradação lenta dos osciladores que compõem essa rede. Assume-se que 


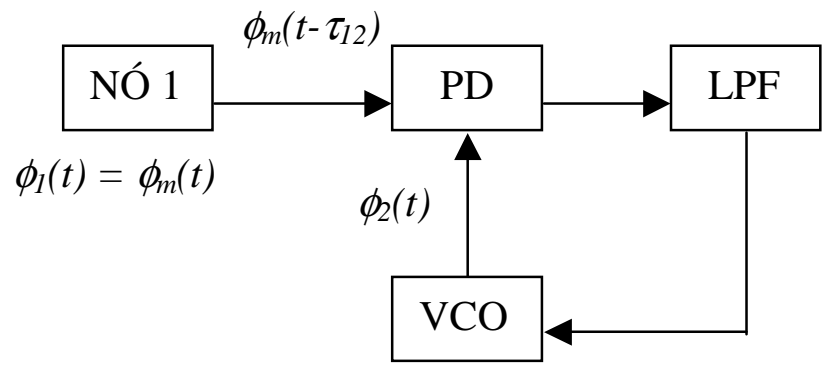

Figura 3: Rede cadeia simples composta de um mestre e um escravo

há um atraso $\tau_{12}$ na propagação do sinal do mestre para o escravo.

O nó mestre é formado por um oscilador cuja freqüência característica vale $\omega_{m}$ e a fase, $\phi_{m}$. A fase do sinal de saída do nó $1, \phi_{1}$, é a própria fase $\phi_{m}$. Ou seja, $\phi_{1}=\phi_{m}$. A fim de se considerar os efeitos da degradação nesse oscilador, assume-se que a variação de $\phi_{m}$ é, na realidade, dada por:

$$
\phi_{m}(t)=\omega_{m} t+\left(\Omega_{m} t^{2}\right) / 2
$$

sendo $\Omega_{m}$ uma componente de aceleração de fase que expressa a degradação do oscilador mestre. Tomar a degradação como um termo proporcional a $t^{2}$ é a maneira analiticamente mais simples de levá-la em conta. O sinal do mestre serve de entrada para o oscilador escravo, após um tempo $\tau_{12}$.

O nó escravo é caracterizado por uma freqüência $\omega_{2}$ e por componente de aceleração $\Omega_{2}$, que reflete a degradação desse oscilador. Considerando que o nó escravo é um PLL de segunda ordem, deduz-se a seguinte equação diferencial para a rede:

$$
\begin{aligned}
& \varphi(t)^{\prime \prime}+ \mu_{1} \varphi(t)^{\prime}+\mu \mu_{1} \operatorname{sen}\left[\phi_{m}\left(t-\tau_{12}\right) \phi_{2}(t)\right]= \\
& \phi_{m}(t)^{\prime \prime}+\mu_{1} \phi_{m}(t)^{\prime}-\Omega_{2}-\mu_{1} \omega_{2}-\left(\mu_{1} \Omega_{2}\right) t
\end{aligned}
$$

sendo $\varphi(t) \equiv \phi_{m}(t)-\phi_{2}(t)$ o erro de fase entre o nó mestre e o nó escravo. Essa dedução é similar àquela feita na seção anterior para um PLL de segunda com entrada $V_{i}$. Por simplicidade, toma-se a aproximação $\phi_{m}\left(t-\tau_{12}\right) \approx \phi_{m}(t)-\tau_{12} d \phi_{m}(t) / d t$, que é válida para atraso "pequeno". Substituindo (3) em (4), obtémse:

$$
\begin{gathered}
\varphi(t)^{\prime \prime}+\mu_{1} \varphi(t)^{\prime}+\mu \mu_{1} \operatorname{sen}\left[\varphi(t)-\tau_{12}\left(\omega_{m}+\Omega_{m} t\right)\right]= \\
\left(\Omega_{m}-\Omega_{2}\right)+\mu_{1}\left(\omega_{m}-\omega_{2}\right)-\mu_{1}\left(\Omega_{m}-\Omega_{2}\right) t
\end{gathered}
$$

A equação (5) possui termos que dependem explicitamente de $t$. Conseqüentemente, tal sistema dinâmico não apresenta pontos de equilíbrio. Assim, o erro de fase $\varphi$ jamais se torna constante, crescendo (em módulo) de maneira ilimitada. Ou seja, a degradação dos osciladores e o atraso na propagação do sinal impedem que o escravo oscile na mesma fase que o mestre, em regime estacionário.

Entretanto, o erro de freqüência $\varphi^{\prime}$ pode se tornar constante, quando sen $x \approx x$. Para analisar essa possibilidade, deriva-se a equação (5) com relação a $t$, levando-se em conta essa aproximação, e chega-se a:

$$
\varphi(t)^{\prime \prime \prime}+\mu_{1} \varphi(t)^{\prime \prime}+\mu \mu_{1}\left[\varphi(t)^{\prime}-\tau_{12} \Omega_{m}\right]=-\mu_{1}\left(\Omega_{m}-\Omega_{2}\right)
$$

Reescrevendo essa equação como um sistema bidimensional de primeira ordem, em termos das variáveis $x \equiv$ $\varphi^{\prime}$ e $y \equiv \varphi$, mostra-se que:

$$
\begin{gathered}
x^{\prime}=y \\
y^{\prime}=-\mu_{1} y-\mu \mu_{1}\left[x-\tau_{12} \Omega_{m}\right]-\mu_{1}\left(\Omega_{m}-\Omega_{2}\right)
\end{gathered}
$$

Esse sistema possui um único ponto de equilíbrio $(x *, y *)=($ constante, 0$)$ que é assintoticamente estável, já que os autovalores da matriz jacobiana associada sempre possuem parte real negativa. Note que $y *=0$ implica $\left(\phi_{m}^{\prime \prime}\right)^{*}=\left(\phi_{2}^{\prime \prime}\right)^{*}$. Como $\phi_{m}^{\prime \prime}=\Omega_{m}$, então, no limite $t \rightarrow \infty$, há uma imposição da aceleração da freqüência do nó mestre sobre o nó escravo.

No caso particular em que são iguais as acelerações de freqüência dos osciladores $\left(\Omega_{m}=\Omega_{2}\right)$ e é nulo o atraso na propagação do sinal $\left(\tau_{12}=0\right)$, então a equação (4) torna-se estruturalmente equivalente à equação (1) com $\mathrm{F}(\mathrm{t})=$ constante, e o diagrama de bifurcação correspondente é equivalente à figura 2 . A bifurcação sela-nó ocorre para $\omega_{m}-\omega_{2}=\mu$.

\section{REDES MESTRE-ESCRAVO ENLACE SIMPLES}

Seja uma rede composta por um mestre e um escravo em topologia enlace simples, como mostra a figura 4 . O nó escravo é representado por um PLL de segunda ordem. O nó mestre é um oscilador de livre curso, que gera o sinal de fase $\phi_{m}$. Sua saída $\phi_{1}$, nessa topologia, é dada por uma combinação linear entre $\phi_{m}$ e o sinal de fase $\phi_{2}$ recebido do nó escravo, após um tempo $\tau_{21}$. Assim:

$$
\phi_{1}(t)=A \phi_{m}(t)-\phi_{2}\left(t-\tau_{21}\right)
$$

sendo $\phi_{m}(t)$ dado pela expressão (3) e $A$ uma constante.

Levando em conta o atraso $\tau_{12}$ na propagação do sinal do mestre para o escravo, mostra-se que a variação 


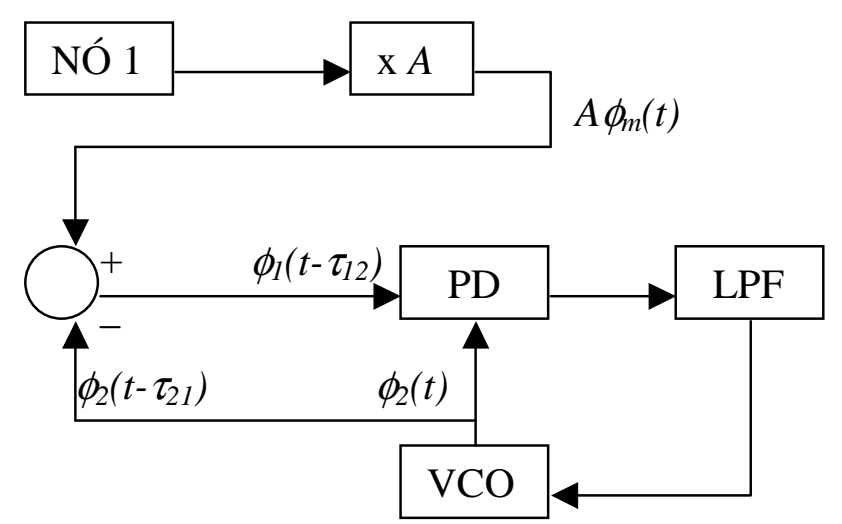

Figura 4: Rede enlace simples composta de um mestre e um escravo

temporal de $\varphi(t) \equiv \phi_{m}(t)-\phi_{2}(t)$ obedece à equação:

$$
\begin{aligned}
\varphi(t)^{\prime \prime}+\mu_{1} \varphi(t)^{\prime}+\mu \mu_{1} \operatorname{sen}\left[\phi_{1}\left(t-\tau_{12}\right)-\phi_{2}(t)\right]= \\
\phi_{m}(t)^{\prime \prime}+\mu_{1} \phi_{m}(t)^{\prime}-\Omega_{2}-\mu_{1} \omega_{2}-\left(\mu_{1} \Omega_{2}\right) t
\end{aligned}
$$

Substituindo as expressões (3) e (8) e tomando a aproximação $\phi(t-\tau) \approx \phi(t)-\tau d \phi(t) / d t$, obtém-se:

$$
\begin{aligned}
& \varphi(t)^{\prime \prime}+\mu_{1} \varphi(t)^{\prime}+\mu \mu_{1} \operatorname{sen}\left[2 \varphi(t)-\left(\tau_{12}+\tau_{21}\right) \varphi(t)^{\prime}+\right. \\
& \left(\omega_{m}+\Omega_{m} t\right)\left[(1-A) \tau_{12}+\tau_{21}\right]+\left(A-2\left(\omega_{m} t+\Omega_{m} t^{2} / 2\right)\right]= \\
& \quad\left(\Omega_{m}-\Omega_{2}\right)+\mu_{1}\left(\omega_{m}-\omega_{2}\right)-\mu_{1}\left(\Omega_{m}-\Omega_{2}\right) t \quad(10)
\end{aligned}
$$

A equação (10) é não-autônoma. Portanto, $\varphi(t)$ não atinge um regime estacionário. Para se verificar se $\varphi(t)^{\prime}$ pode se tornar constante, no limite sen $x \approx x$, deriva-se (10), obtendo:

$$
\begin{array}{r}
\varphi(t)^{\prime \prime \prime}+\mu_{1} \varphi(t)^{\prime \prime}+\mu \mu_{1}\left[2 \varphi(t)^{\prime}-\left(\tau_{12}+\tau 21\right) \varphi(t)^{\prime \prime}+\right. \\
\Omega_{m}\left[(1-A) \tau_{12}+\tau_{21}\right]+\left(A-2\left(\omega_{m}+\Omega_{m} t\right)\right] \\
=-\mu_{1}\left(\Omega_{m}-\Omega_{2}\right)
\end{array}
$$

Repare que para $A=2$, o termo que depende explicitamente do tempo se anula. Cideciyan e Lindsey (1987), ao analisarem essa rede, assumiram, de saída, $A=2$, sem, contudo, declararem o motivo dessa escolha.

Reescrevendo a equação (11) para $A=2$ como um sistema bidimensional de primeira ordem em termos das variáveis $x \equiv \varphi^{\prime}$ e $y \equiv \varphi^{\prime \prime}$, como foi feito no caso anterior, mostra-se que esse sistema possui um único ponto de equilíbrio $(x *, y *)=($ constante, 0$)$, que é assintoticamente estável somente se $\left(\tau_{12}+\tau_{21}\right)<1 / \mu$. Como na rede cadeia simples, ocorre uma imposição da aceleração da frequiência do nó mestre sobre o nó escravo.

No caso particular em que são iguais as acelerações de freqüência dos osciladores $\left(\Omega_{m}=\Omega_{2}\right)$, são iguais os atra$\operatorname{sos} \tau_{12}=\tau_{21}=\tau$ e $\mathrm{A}=2$, a equação (10) torna-se:

$\varphi(t)^{\prime \prime}+\mu_{1} \varphi(t)^{\prime}+\mu \mu_{1} \operatorname{sen}\left[2 \varphi(t)-2 \tau t \varphi(t)^{\prime}\right]=\mu_{1}\left(\omega_{m}-\omega_{2}\right)$

que admite uma solução de equilíbrio $\varphi^{*}$ assintoticamente estável somente se $\tau<$ $1 /\left(2 \mu \cos 2 \varphi^{*}\right)$ e $\cos 2 \varphi^{*}>0$. Análises de sistemas não-lineares não foram realizadas por Cideciyan e Lindsey (1987).

\section{CONCLUSÕES}

Neste trabalho, utiliza-se um método, baseado na Teoria de Sistemas Dinâmicos, para se analisar sincronismo em redes de osciladores acoplados. Esse método consiste de: obter a equação diferencial que governa a variação da diferença de fases entre dois osciladores adjacentes; reescrever tal equação como um conjunto de equações diferenciais de primeira ordem; e determinar a existência e a estabilidade da solução síncrona.

As redes mestre-escravo, aqui analisadas, apresentam soluções síncronas apenas para certas combinações de valores dos parâmetros. Na topologia cadeia simples, tal solução só existe no caso em que o atraso $\tau_{12}$ é nulo e as acelerações nas fases do mestre e do escravo, causadas pela degradação de seus osciladores, são iguais $\left(\Omega_{m}\right.$ $=\Omega_{2}$ ). Na topologia enlace simples, é necessário que $\Omega_{m}=\Omega_{2}, \tau_{12}=\tau_{21}$ e $A=2$. Para outras combinações de valores dos parâmetros, o erro de freqüência entre o mestre e o escravo pode tornar-se limitado, quando é válida a aproximação sen $x \approx x$, realizada para a obtenção das expressões (6) e (11).

Note que um atraso, mesmo se "pequeno", restringe fortemente a existência de soluções síncronas em cadeias de osciladores. Por isso, seu efeito deve ser compensado, quando busca-se sincronismo. A análise de técnicas de compensação de atraso, à luz da Teoria de Sistemas Dinâmicos, será assunto de trabalhos futuros.

Configurações topologicamente mais intrincadas podem, em princípio, ser analisadas através do método aqui utilizado.

\section{REFERÊNCIAS}

Bernardo D., Signorini M.G. e Cerutti S. (1998). A Model for Two Nonlinear Coupled Oscillators for the Study of Heartbeat Dynamics. Int. J. Bifurcation Chaos 8: 1975-1985.

Buck J. e Buck E. (1976). Synchronous Fireflies. Sci. 
Am. 234(5): 74-85.

Cideciyan R.D. e Lindsey W.C. (1987). Effects of longterm instability on master-slave networks. IEEE Trans. Commun. 35: 950-955.

Cohen A.H., Holmes P.J. e Rand R.H. (1982). The nature of the coupling between segmental oscillators of the lamprey spinal generator for locomotion: a mathematical model. J. Math. Biol. 13: 345-369.

Engel A.K., König P., Kreiter A.K., Schillen T.B. e Singer W. (1992). Temporal Coding in the Visual Cortex: New Vistas on Integration in the Nervous System. Trends Neurosci. 15: 218-226.

Garcia, P.A. (2000). Redes Simples de Malhas de Sincronismo de Fase: uma Análise via Teoria dos Sistemas Dinâmicos. Dissertação de Mestrado. Pósgraduação em Engenharia Elétrica da Universidade Presbiteriana Mackenzie.

Gersho A. e Karafin B.J. (1966). Mutual Synchronization of Geographically Separated Oscillators. Bell Sist. Tech. J. 45: 1689-1704.

Guckenheimer J. e Holmes P. (1983). Nonlinear Oscillations, Dynamical Systems, and Bifurcations of Vector Fields. New York: Springer.

Hsieh G.C. e Hung J.C. (1996). Phase-locked Loop Techniques - a Survey. IEEE Trans. Ind. Electron. 43: 609-615.

Karnaugh M. (1966). A Model for Organic Synchronization of Communication Systems. Bell Syst. Tech. J. 45: 1705-1735.

Lindsey W.C., Ghazvinian F., Hagmann W.C. e Dessouky K. (1985). Network synchronization. Proc. IEEE 73: 1445-1467.

McClintock M.K. (1971). Menstrual Synchrony and Suppression. Nature 229: 244-245.

Monteiro L.H.A. (2002). Sistemas Dinâmicos. São Paulo: Livraria da Física.

Néda Z., Ravasz E., Vicsek T., Brechet Y. e Barabási A.L. (2000). Physics of the Rhythmic Applause. Phys. Rev. E. 61: 6987-6992.

Piqueira J.R.C. (1997). Uma Contribuição ao Estudo das Redes de Malhas de Sincronismo de Fase. Tese de Livre-docência. Escola Politécnica da Universidade de São Paulo.
Sexton M.J. e Reid A.B. (1992). Transmission Networking: SONET and the Synchronous Digital Hierarchy. London: Artech House.

Walker T.J. (1969). Acoustic Synchrony: Two Mechanisms in the Snowy Tree Cricket. Science 166: 891894. 